\title{
General power-law temporal scaling for unequal-size microbubble coalescence
}

\author{
Rou Chen $\odot$, Huidan (Whitney) Yu, ${ }^{*}$ Jianhuan Zeng, and Likun Zhu \\ Mechanical \& Energy Engineering Department, Indiana University-Purdue University, Indianapolis (IUPUI), Indiana, 46202, USA
}

(Received 10 December 2018; revised manuscript received 8 July 2019; accepted 13 January 2020; published 10 February 2020)

\begin{abstract}
We systematically study the effects of liquid viscosity, liquid density, and surface tension on global microbubble coalescence using lattice Boltzmann simulation. The liquid-gas system is characterized by Ohnesorge number Oh $\equiv \eta_{h} / \sqrt{\rho_{h} \sigma r_{F}}$ with $\eta_{h}, \rho_{h}, \sigma$, and $r_{F}$ being viscosity and density of liquid, surface tension, and the radius of the larger parent bubble, respectively. This study focuses on the microbubble coalescence without oscillation in an Oh range between 0.5 and 1.0. The global coalescence time is defined as the time period from initially two parent bubbles touching to finally one child bubble when its half-vertical axis reaches above 99\% of the bubble radius. Comprehensive graphics processing unit parallelization, convergence check, and validation are carried out to ensure the physical accuracy and computational efficiency. From 138 simulations of 23 cases, we derive and validate a general power-law temporal scaling $T^{*}=A_{0} \gamma^{-n}$, that correlates the normalized global coalescence time $\left(T^{*}\right)$ with size inequality $(\gamma)$ of initial parent bubbles. We found that the prefactor $A_{0}$ is linear to $\mathrm{Oh}$ in the full considered Oh range, whereas the power index $n$ is linear to Oh when $\mathrm{Oh}<0.66$ and remains constant when $\mathrm{Oh}>0.66$. The physical insights of the coalescence behavior are explored. Such a general temporal scaling of global microbubble coalescence on size inequality may provide useful guidance for the design, development, and optimization of microfluidic systems for various applications.
\end{abstract}

DOI: 10.1103/PhysRevE.101.023106

\section{INTRODUCTION}

Microbubble coalescence is referred to as an evolving process during which two or more touching bubbles (parents), with their diameters from 1-100 $\mu \mathrm{m}$, merge to a single bubble (child). A global coalescence typically consists of two stages. The first one is the early coalescence during which neck bridges form and grow. The second one is the post coalescence toward a child bubble with a minimal surface area. Microbubble coalescence exists in many applications, such as airlift bioreactors [1], targeted drug and gene delivery [2], water and wastewater treatment [3], and food storage [4]. In some cases, rapid coalescence might be desirable. One example is to control bubble formation during gas injection from a microtube into the channel of a downward liquid cross flow [5]. While in other systems, coalescence needs to be prevented or suppressed to avoid the loss of the total liquid-gas surface area. Therefore, it is of general interest to explore the dynamics of microbubble coalescence under various influences for better control of various gas-fluid systems.

There have been efforts to investigate different effects on the individual stages of microbubble coalescence through mathematical analyses, laboratory experiments, and numerical simulations. The majority efforts have been on the early coalescence to reveal various effects [6-10]. For instance, Paulsen et al. [8] studied the effects of the dense surrounding fluid on the formation of an infinitesimal neck bridge and discovered that outer fluid has a marginal impact on the dynamics

\footnotetext{
*whyu@iupui.edu
}

of neck bridge formation and evolution. On this early stage, a half power-law temporal scaling of neck growth has been well derived and validated. However, the post-coalescence stage has been rarely addressed. An early attempt was on pure mathematical modeling for an oscillating ellipse [11]. The corresponding numerical simulations were done [12] after two decades under characterized parameters. Based on these mathematical and numerical frameworks, Stover et al. [13] experimentally studied the microbubble oscillation in the post coalescence stage. This study focused on the effects of liquid viscosity and surface tension on the decay of the damped oscillation but lacked a quantitative exploration of the underlying physics of the oscillation. It is substantially meaningful to understand the post coalescence because very different behavior could occur in this coalescence stage such as damped oscillation [13,14], shrinking [15], and off-center or head-on separation [16]. However, there remains a challenge to study global coalescence, especially when size inequality of parent bubbles is involved.

The motivation of this study stemmed from an experiment to prototype and fabricate a novel microreactor for on-chip gas generation [17]. The major behavior of this microfluidic device is unequal-size bubble coalescence [18]. We explore the underlying physics in the global process of microbubble coalescence to support the design and optimization of the prototype. Prior to this work, we have studied the temporal and spatial scalings of air microbubble coalescence in water [19], the effects of the initial conditions on the neck growth [9] in the early coalescence, and the mechanism of damping oscillation in the post coalescence [14] through numerical simulations using the lattice Boltzmann method (LBM) $[20,21]$. 
We showed that unequal-size air microbubble coalescence in water exhibits a power-law temporal scaling [19]. We also learned that an Ohnesorge number (Oh, defined in Sec. III A) determines whether a damping oscillation would occur in the post-coalescence stage [14]. We have identified a critical Oh value $(\approx 0.477)$ that separates two distinct post-coalescence behaviors: with or without damping oscillation when $\mathrm{Oh}$ is smaller or larger than 0.477 , respectively. The mechanism behind the damping oscillation has been explored in terms of the competition between driving and resisting forces in the two fluids.

In this study, we further investigate the general effects of inertia and viscosity on the power-law temporal scaling of global bubble coalescence when the Oh number is relatively large, i.e., $\mathrm{Oh}>0.5$. In this range of $\mathrm{Oh}$, no damping oscillation occurs in the post-coalescence stage. Totally 23 cases, characterized by $\mathrm{Oh}$ number with different combinations of liquid density, viscosity, and surface tension, are systematically studied and a general power-law temporal scaling is derived and validated for unequal-size microbubble coalescence. We continue to employ the multiphase LBM model [22-25] based on the free-energy theory [26-28]. This model has been continuously developed and refined by Lee's group [29-32]. In this model, the parasitic current (a smallamplitude artificial velocity field arising from an imbalance between discretized forces in multiphase and multicomponent flows) has been eliminated. It is noted that minimizing the parasitic current is critically important for accurately simulating multiphase flows.

The computation cost for simulating the global coalescence of microbubbles is high. A global microbubble coalescence typically takes about 300 milliseconds. With appropriate temporal and spatial resolutions, one complete simulation through a serial execution on a workstation (Intel@ Xeon@ CPU X5660@2.80 GHz) takes about151 wall-clock hours. For the 138 simulations in the 23 cases in this study, the total computation time would be approximately 2.5 years. Using LBM with parallel computing can overcome such a computation bottleneck. It has been well known that the LBM is a versatile and highly parallel approach as the discrete Boltzmann transport equation is solved in the velocity (or moment) space to obtain the time-dependent fluid velocity distributions. Due to the intrinsic parallel nature, the implementation of LBM on graphics processing units (GPUs) has become increasingly popular [33,34]. Vanka group [35,36] was among the early groups to study the GPU acceleration for simulating complex flows including turbulence and multiphase flow. They got around 25 times speedup over a single CPU $(2.6 \mathrm{GHz}$ AMD Phenom quad-core processor) performance through a single-core GPU (Tesla C2070, CUDA 3.2 compiler) implementation. With the recent fast development of both CPU and GPU hardware, as well as computation algorithms, the speedup ratio of GPU parallel vs CPU serial computation for multiphase flows using LBM has been consistently increasing. Most recently, the acceleration ratio of GPU (Tesla P100) over CPU (i7-4930K) performance has reached 680 MLUPS (million lattice updates per second), using LBM to simulate droplet dynamics [37]. Since 2013, we have been implementing GPU parallel computing in different research projects, such as direct numerical simulation of decaying isotropic tur- bulence [38], patient-specific computational hemodynamics [39], image-based pore-scale porous media flows [40,41], and the current multiphase flow. With about 60 times speedup using GPU parallel (Tesla C2075) computation, we have reduced the wall-clock hours from 2.5 years, as aforementioned, to 14.4 days, making it possible to study over 23 cases (138 simulations) within a manageable time. Using the computation resources of XSEDE [42], we achieved 981 times speedup of GPU parallel (Tesla P100 GPU) from CPU serial (Intel Broadwell, E5-2683 v4) computation. A corresponding manuscript addressing the GPU parallelism and acceleration is in preparation.

The remainder of the paper is organized as follows. Section II shows the mathematical formulation of lattice Boltzmann modeling for fluid-gas flows. The computational setup, validation, and numerical results are presented in Sec. III. Finally, Sec. IV provides a summary discussion and concludes the paper.

\section{LATTICE BOLTZMANN MODELING FOR FLUID-GAS FLOWS}

We concisely introduce the main modeling idea and major equations here. The detailed formulation of the lattice Boltzmann model can be found in [19]. When the flow involves both fluid and gas, the interfacial behavior arises as a result of microscopic long-range interactions among the constituent molecules of the system [43]. Using a diffuse interface to separate phases is a popular technique in the modeling of multiphase flow. The advantages include the ease of implementation (even for complex three-dimensional interfaces) and the suitability to capture singular phenomena, such as interface rupture, coalescence, or phase change. The governing equations including Cahn-Hilliard equation, pressure evolution equation, and momentum equation are

$$
\begin{gathered}
\partial C / \partial t+\mathbf{u} \cdot \nabla C=\nabla \cdot(M \nabla \mu) \\
\partial p_{1} / \partial t+\rho c_{s}^{2} \nabla \cdot \mathbf{u}=0 \\
\rho(\partial \mathbf{u} / \partial t+\mathbf{u} \cdot \nabla \mathbf{u})=-\nabla p_{1}+\mu \nabla C+\nabla \cdot \eta\left(\nabla \mathbf{u}+(\nabla \mathbf{u})^{T}\right) .
\end{gathered}
$$

In the equations, $C\left(=\tilde{\rho}_{i} / \rho_{i}\right)$ is the composition of liquid and gas, $\mu$ is the chemical potential with $\mu=\mu_{0}-\kappa \nabla^{2} C$ in which $\mu_{0}$ is the classical part of the chemical potential. We assume that the energy $E_{0}$ takes a form $[43,44]$ of $E_{0}=$ $\beta C^{2}(C-1)^{2}$ with $\beta$ being a constant. Thus, $\mu_{0}=\partial E_{0} / \partial C=$ $2 \beta C(C-1)(2 C-1)$.

The equilibrium profile of $C$ is determined when the energy $E_{0}$ is minimized and $\mu$ is a constant in one dimension. At equilibrium, the interface profile is $C(z)=0.5+0.5 \tanh (2 z / D)$ where $z$ is the distance normal to the interface and $D$ is the (numerical) interface thickness. The gradient parameter $\kappa=\beta D^{2} / 8$ and the surface tension $\sigma=\sqrt{2 \kappa \beta} / 6$ can be calculated when $D$ and $\beta$ are given. The intermolecular force [31] is $\mathbf{F}=\frac{1}{3} \nabla \rho c^{2}-\nabla p_{1}-C \nabla \mu$ where $p_{1}$ is the hydrodynamic pressure. The thermodynamic pressure $p_{0}$ is defined by $p_{0}=$ $C \partial E_{0} / \partial C-E_{0}=\beta C^{2}(C-1)(3 C-1)$. The total pressure is $p=p_{0}+p_{1}-\kappa C \nabla^{2} C+\kappa|\nabla C|^{2} / 2$. 
The lattice Boltzmann equation (LBE) (before the time discretization) including the intermolecular force is [24]

$$
\partial f_{\alpha} / \partial t+\mathbf{e}_{\alpha} \cdot \nabla f_{\alpha}=-\left(f_{\alpha}-f_{\alpha}^{e q}\right) / \lambda+\frac{3}{c^{2}}\left(\mathbf{e}_{\alpha}-\mathbf{u}\right) \cdot \mathbf{F} f_{\alpha}^{e q},
$$

where $f_{\alpha}$ is the particle distribution function with discrete molecular velocity $\mathbf{e}_{\alpha}$ along the $\alpha$ th direction and $\lambda$ is the relaxation time related to the kinematic viscosity $\nu=\frac{1}{3} c^{2} \lambda$. The equilibrium distribution function, a function of local macroscopic density and velocity, is usually formulated up to $O\left(u^{2}\right)$, i.e., $f_{\alpha}^{e q}=\rho \omega_{\alpha}\left[1+3\left(\mathbf{e}_{\alpha} \cdot \mathbf{u}\right) / c^{2}+9\left(\mathbf{e}_{\alpha} \cdot \mathbf{u}\right)^{2} /\left(2 c^{4}\right)-\right.$ $\left.3 \mathbf{u}^{2} /\left(2 c^{2}\right)\right]$. In the formula, $\omega_{\alpha}$ is the weight associated with a particular discretized velocity $\mathbf{e}_{\alpha}, \rho$ and $\mathbf{u}$ are macroscopic density and velocity, respectively, and $c=\delta x / \delta t=1$ in lattice units (i.e., $\delta t=\delta x=1$ ).

In order to recover the second and the third governing equations, i.e., Eqs. (2) and (3), we introduce a new distribution function $g_{\alpha}=\frac{1}{3} f_{\alpha} c^{2}+\left(p_{1}-\frac{1}{3} \rho c^{2}\right) \Gamma_{\alpha}(0)$, in which $\Gamma_{\alpha}(\mathbf{u})=f_{\alpha}^{e q} / \rho$. Taking the total derivative $D_{t}=\partial_{t}+\mathbf{e}_{\alpha} \cdot \nabla$ of $g_{\alpha}$ results in

$$
\begin{aligned}
\partial g_{\alpha} / \partial t+\mathbf{e}_{\alpha} \cdot \nabla g_{\alpha}= & -\left(g_{\alpha}-g_{\alpha}^{e q}\right) / \lambda+\left(\mathbf{e}_{\alpha}-\mathbf{u}\right) \cdot\left\{\frac { 1 } { 3 } \nabla \rho c ^ { 2 } \left[\Gamma_{\alpha}\right.\right. \\
& \left.\left.-\Gamma_{\alpha}(0)\right]-C \nabla \mu \Gamma_{\alpha}\right\},
\end{aligned}
$$

where the corresponding equilibrium distribution function is $g_{\alpha}^{e q}=\omega_{\alpha}\left\{p_{1}+\rho\left[(\mathbf{e} \cdot \mathbf{u})+3\left(\mathbf{e}_{\alpha} \cdot \mathbf{u}\right)^{2} / 2 c^{2}-\mathbf{u}^{2}\right]\right\}$.

Discretizing Eq. (5) along characteristics over the time step $\delta t$, we obtain the LBE for $g_{\alpha}$ as follows:

$$
\begin{aligned}
\bar{g}_{\alpha}\left(\mathbf{x}+\mathbf{e}_{\alpha} \delta t, t+\delta t\right) \\
=\bar{g}_{\alpha}(\mathbf{x}, t)-\left.\frac{1}{\tau+0.5}\left(\bar{g}_{\alpha}-\bar{g}_{\alpha}^{e q}\right)\right|_{(\mathbf{x}, t)}+\left(\mathbf{e}_{\alpha}-\mathbf{u}\right) \\
\left.\cdot\left\{\frac{1}{3} \delta t \nabla^{\mathrm{MD}} \rho c^{2}\left[\Gamma_{\alpha}(\mathbf{u})-\Gamma_{\alpha}(0)\right]-C \delta t \nabla^{\mathrm{MD}} \mu \Gamma_{\alpha}\right\}\right|_{(\mathbf{x}, t)} .
\end{aligned}
$$

In Eq. (6), $\nabla^{\mathrm{MD}}\left(=0.5 \nabla^{\mathrm{BD}}+0.5 \nabla^{\mathrm{CD}}\right)$ is referred to mixed difference approximation which presents the average of biased $\left(\nabla^{\mathrm{BD}}\right)$ and central $\left(\nabla^{\mathrm{CD}}\right)$ difference approximation [32], $\tau(=\lambda / \delta t)$ is the dimensionless relaxation time, and $\bar{g}_{\alpha}$ and $\bar{g}_{\alpha}^{e q}$ are introduced below, respectively:

$$
\begin{aligned}
\bar{g}_{\alpha}= & g_{\alpha}+\frac{1}{2 \tau}\left(g_{\alpha}-g_{\alpha}^{e q}\right)-\frac{1}{2} \delta t\left(\mathbf{e}_{\alpha}-\mathbf{u}\right) \\
& \cdot\left\{\frac{1}{3} \nabla^{\mathrm{CD}} \rho c^{2} C\left[\Gamma_{\alpha}(\mathbf{u})-\Gamma_{\alpha}(0)\right]-C \nabla^{\mathrm{CD}} \mu \Gamma_{\alpha}\right\}, \\
\bar{g}_{\alpha}^{e q}= & g_{\alpha}^{e q}-\frac{1}{2} \delta t\left(\mathbf{e}_{\alpha}-\mathbf{u}\right) \\
& \cdot\left\{\frac{1}{3} \nabla^{\mathrm{CD}} \rho c^{2}\left[\Gamma_{\alpha}(\mathbf{u})-\Gamma_{\alpha}(0)\right]-C \nabla^{\mathrm{CD}} \mu \Gamma_{\alpha}\right\} .
\end{aligned}
$$

The momentum and hydrodynamic pressures of the liquid-gas system are the zeroth and first-order moments of $\bar{g}_{\alpha}$. They are computed as $\rho u=\frac{3}{c^{2}} \sum \mathbf{e}_{\alpha} \bar{g}_{\alpha}-\frac{\delta t}{2} C \nabla^{\mathrm{CD}} \mu$ and $p_{1}=\sum \bar{g}_{\alpha}+$ $\frac{\delta t}{6} \mathbf{u} \cdot \nabla^{\mathrm{CD}} \rho c^{2}$.

To recover the first governing equation of Eq. (1), we introduce another new distribution function $h_{\alpha}=(C / \rho) f_{\alpha}$ with

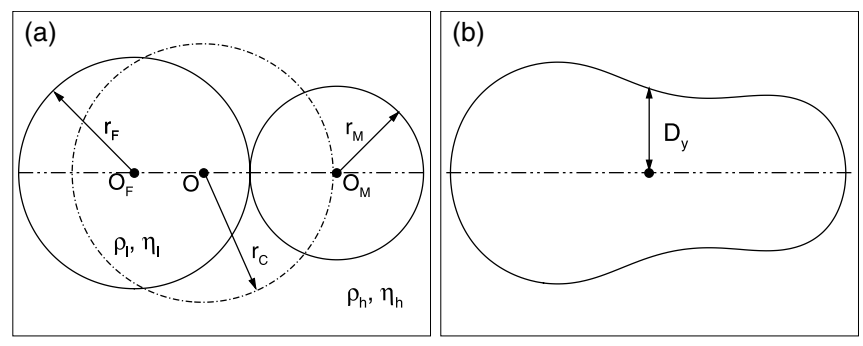

FIG. 1. Schematic diagrams for a global coalescence of two unequal-size microbubbles in a square domain periodic in both directions. (a) Initial parent bubbles (solid circles) and final child bubble (dashed-dotted circle). (b) A coalescing child bubble with $D_{y}$ defined as the half-vertical axis at the center of horizontal axis.

its equilibrium distribution function being $h_{\alpha}^{e q}=(C / \rho) f_{\alpha}^{e q}$. Similarly, taking the total derivative $D_{t}$ of $h_{\alpha}$ and utilizing Eq. (1) yields

$$
\begin{aligned}
\bar{h}_{\alpha}(\mathbf{x}+ & \left.\mathbf{e}_{\alpha} \delta t, t+\delta t\right) \\
= & \bar{h}_{\alpha}(\mathbf{x}, t)-\frac{\left.\left(\bar{h}_{\alpha}-\bar{h}_{\alpha}^{e q}\right)\right|_{(\mathbf{x}, t)}}{\tau+0.5}+\delta t\left\{\left(\mathbf{e}_{\alpha}-\mathbf{u}\right)\right. \\
& \cdot\left[\nabla^{\mathrm{MD}} C-\frac{3 C}{\rho c^{2}}\left(\nabla^{\mathrm{MD}} p_{1}+C \nabla^{\mathrm{MD}} \mu\right)\right] \\
& \left.+M \nabla^{2} \mu\right\}\left.\Gamma_{\alpha}\right|_{(\mathbf{x}, t)} .
\end{aligned}
$$

In Eq. (9), $\bar{h}_{\alpha}$ and $\bar{h}_{\alpha}^{e q}$ are defined as the same formats as $\bar{g}_{\alpha}$ and $\bar{g}_{\alpha}^{e q}$ [32], respectively.

The composition $C$ is the zeroth-order moment of $\bar{h}_{\alpha}$, obtained by $C=\sum_{\alpha} \bar{h}_{\alpha}+0.5 \delta t M \nabla^{2} \mu$. The density $\rho$ and the dimensionless relaxation frequency $(1 / \tau)$ are linear functions of the composition, given by $\rho(C)=C \rho_{1}+(1-C) \rho_{2}$ and $1 / \tau(C)=C / \tau_{1}+(1-C) / \tau_{2}$.

\section{NUMERICAL STUDY}

The objective of this numerical study below is to address two pertinent questions. (1) If the power-law temporal scaling of unequal-size microbubble coalescence [19] is general? (2) How is the power-law temporal scaling affected by inertia, viscosity, and surface tension?

\section{A. Computation setup and GPU parallelism}

We consider two unequal-size microbubbles coalesce in a square domain of $100^{2} \mu \mathrm{m}^{2}$ with periodic boundary in each direction. As schematized in Fig. 1(a), the center of the father bubble, $O_{F}$, is located at $x=30 \mu \mathrm{m}$ and $y=50 \mu \mathrm{m}$ with a fixed radius $r_{F}=20 \mu \mathrm{m}$. The mother bubble is attached to the father bubble on the right, of which the center is aligned at the same vertical location. The radius of the mother bubble varies from 5 to $20 \mu \mathrm{m}$, resulting in the size inequality $\gamma\left(\equiv r_{F} / r_{M}\right)$ in the range of 4 to 1 . Thus, the center of the mother bubble, $O_{M}$, is located at $x=50+r_{M}(\mu \mathrm{m})$ and $\mathrm{y}=50 \mu \mathrm{m}$. The radius of the final child bubble (dashed-dotted circle), $r_{C}$, can be determined by the total area of the parent bubbles. Figure 1(b) depicts an intermediate stage of the coalescing 
TABLE I. GPU acceleration comparing GPU parallel with CPU serial performance for simulating a global microbubble coalescence. The last column shows the wall-clock time in hours of GPU parallel and CPU serial computation. MLUPS stands for million lattice updates per second. Hardware: Intel(R) Xeon(R) CPU X5660 @ 2.80 GHz with one Tesla C2075 GPU cards.

\begin{tabular}{lcccc}
\hline \hline Resolution & GPU parallel (MLUPS) & Serial (MLUPS) & Speedup ratio of parallel vs Serial & Parallel/Serial (in hours) \\
\hline $200 \times 200$ & 15.5 & 0.479 & 32.4 & $0.25 / 8.0$ \\
$400 \times 400$ & 17.5 & 0.422 & 41.5 & $1.2 / 49.8$ \\
$600 \times 600$ & 19.9 & 0.396 & 50.2 & $3.0 / 151.2$ \\
$800 \times 800$ & 20.9 & 0.378 & 55.2 & $6.0 / 331.2$ \\
$1000 \times 1000$ & 21.3 & 0.367 & 58.2 & $10.0 / 583.2$ \\
\hline \hline
\end{tabular}

child bubble with $D_{y}$ defined as the half-vertical axis at the center of horizontal axis. The global coalescence time $T$ is defined as the time period from two parent microbubbles initially touching to finally one coalesced child bubble. The end time point of $T$ is determined when $\left|D_{y}-r_{C}\right| / r_{C}<1 \%$. It is noted that without oscillation in the post coalescence, $D_{y}$ asymptotically increases toward $r_{C}$ during the coalescence. Subscripts of " $h$ " and "l" denote the heavy (liquid) and light (gas) fluid, respectively. The density and viscosity ratios of two fluids are defined as $\rho^{*}=\rho_{h} / \rho_{l}$ and $\eta^{*}=\eta_{h} / \eta_{l}$, respectively. We define the aforementioned Oh number as $\mathrm{Oh} \equiv$ $\eta_{h} / \sqrt{\rho_{h} \sigma r_{F}}$. The Oh effects on drop coalescence have been indicated in a review paper [45] as follows: when $\mathrm{Oh} \ll 1$, the coalescence is dominated by the inertial force and the viscous effect is insignificant; when Oh is of order unity, the coalescence is dominated by viscous force and the inertial effect becomes insignificant. For these two ends in the $\mathrm{Oh}$ spectrum, two characteristic timescales have been introduced [46]: $t_{i}=\sqrt{\rho_{h} r_{F}^{3} / \sigma}$ and $t_{v}=\eta_{h} r_{F} / \sigma$ as inertial and viscous timescale, respectively. In between, i.e., $0.2<\mathrm{Oh}<1$, both inertial and viscous forces contribute to the resisting force in the coalescence process and $t_{i}$ and $t_{v}$ have approximately the same order. Thus, either $t_{i}$ or $t_{v}$ can be used to characterize the coalescence time.

We first develop GPU parallel computing for the LBM code developed in our previous study $[14,19]$. Based on the previous CUDA-GPU implementation of LBM for several single-phase flows [38-41], we employ the same CUDA parallel algorithm in the current two-phase flow. The parallelism is carried out on Intel $($ Xeon $($ CPU X5660@ $2.80 \mathrm{GHz}$ with one Tesla C2075 GPU card. Table I shows the comparison between CPU serial and GPU parallel computation with five spatial resolutions from $200^{2}$ to $1000^{2}$ for simulating a global microbubble coalescence. As a standard measure in parallel LBM, we use MLUPS to represent the computation performance. The fourth column shows the performance ratio of GPU parallel to CPU serial computation. It is seen that the GPU acceleration increases when the resolution increases. For the resolution of $800 \times 800$, which is used in this study, the wall-clock time is reduced from $331.2 \mathrm{~h}$ (CPU serial computing) to $6 \mathrm{~h}$ (GPU parallel commutating). Although the acceleration is less significant than what is achieved in our other projects for single-phase flows [38,40,41], 50 times speedup has significantly enhanced the computation efficiency for the massive parametric simulations in this study. With the optimization of the parallel algorithm and the involvement of multiple GPU card, higher computation efficiency is expected in the near future.

\section{B. Convergence check and validation}

In order to find out an appropriate spatial resolution for the parametric study, we select a case with $\mathrm{Oh}=0.509$ to conduct a convergence check. The physical quantities are $\rho_{h}=$ $896 \mathrm{~kg} / \mathrm{m}^{3}, \rho_{l}=1.28 \mathrm{~kg} / \mathrm{m}^{3}, \eta_{h}=1.22 \times 10^{-3}[\mathrm{~kg} /(\mathrm{m} \mathrm{s})]$, $\eta_{l}=1.74 \times 10^{-5}[\mathrm{~kg} /(\mathrm{ms})]$, and $\sigma=3.2 \times 10^{-2} \mathrm{~N} / \mathrm{m}$. We checked five resolutions from $500 \times 500$ to $900 \times 900$, as seen in Table II. The relative error is calculated from the ratio of the time difference between two successive resolutions and the time of coarse resolution. Since the relative difference between the last two resolutions is smaller than $1 \%$, we select $800 \times 800$ as the spatial resolution to conduct all the simulations discussed below.

Next, we show the reliability of the LBM simulation. The time evolution of the half-vertical axis $D_{y}$ is tracked when two equal-size bubbles are coalescing, corresponding to the neck growth stage [see Fig. 2(a)]. Figure 2(b) shows the development of normalized half-vertical axis $D_{y} / r_{F}$ with normalized time $t / t_{i}$. The symbols are obtained from the numerical simulation. A linear fitting (solid line) for the symbols on a log-log scale results in $D_{y} / r_{F}=1.35\left(t / t_{i}\right)^{1 / 2}$ with the $R$-squared value $R^{2}=0.998$. This result demonstrates that the half power-law scaling of neck growth [6] has been well captured by the current simulation.

TABLE II. Convergence check through a case with $\mathrm{Oh}=0.509$. The global coalescence is simulated using five resolutions from $500^{2}$ to $900^{2}$. The relative difference is calculated from the ratio of the time difference between two successive resolutions to the time of the coarse resolution.

\begin{tabular}{|c|c|c|c|c|c|}
\hline Mesh & $500 \times 500$ & $600 \times 600$ & $700 \times 700$ & $800 \times 800$ & $900 \times 900$ \\
\hline Coalescence time $(\mu \mathrm{s})$ & 87.80 & 83.78 & 81.56 & 80.11 & 79.33 \\
\hline Relative difference & & $4.58 \%$ & $2.65 \%$ & $1.78 \%$ & $0.98 \%$ \\
\hline
\end{tabular}




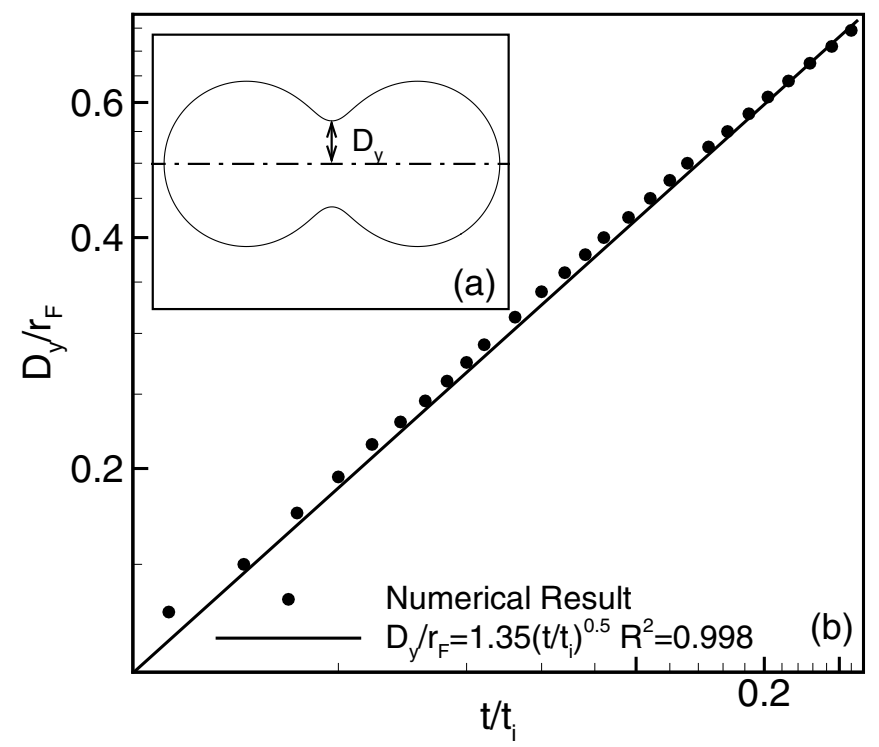

FIG. 2. (a) Illustration of the half-vertical axis $\left(D_{y}\right)$ defined as the vertical distance from the bubble center to the top interface of the bubble. (b) Simulation results (symbols) are well aligned on the half power-law scaling (line) from analytical prediction [6], demonstrating the validity of the LBM simulation.

\section{Numerical results}

The numerical results are on unequal-size microbubbles coalescence in various liquid-gas systems with relatively large Oh numbers $(\mathrm{Oh}>0.447)$, with which no damping oscillation is involved in the post coalescence. We intend to find out if the power-law temporal scaling for air microbubble coalescence in water [19] is general and how the fluid properties affect the scaling.

We fix the density and viscosity of the gas phase as $\rho_{l}=1.28 \mathrm{~kg} / \mathrm{m}^{3}$ and $\eta_{l}=1.74 \times 10^{-5}[\mathrm{~kg} /(\mathrm{m} \mathrm{s})]$ and surface tension as $0.032 \mathrm{~N} / \mathrm{m}$. As shown in Table III, variation of the liquid density $\left(\rho_{h}\right)$ and liquid viscosity $\left(\eta_{h}\right)$ are from 448 to $1482 \mathrm{~kg} / \mathrm{m}^{3}$ and from 0.0111 to $0.0237[\mathrm{~kg} /(\mathrm{m} \mathrm{s})]$, respectively. And the $\mathrm{Oh}$ numbers of the 12 cases range from $0.509 \sim 0.946$. The size inequalities of the parent microbubbles are chosen as $\gamma=4,3,2,1.5,1.2,1$, in which $\gamma=1$ corresponds to the equal-size bubble case. Again, we use the inertial timescale to normalize the global coalescence time as $T^{*}=T / t_{i}$.

Figure 3 shows the numerical result of normalized global coalescence time $T^{*}$ as a function of size inequality $\gamma$ with three different $\mathrm{Oh}$ numbers. In each case, power-law temporal scaling $T^{*}=A_{0} \gamma^{-n}$ (fitting line) is exhibited. The average differences and the correlation coefficients of the numerical

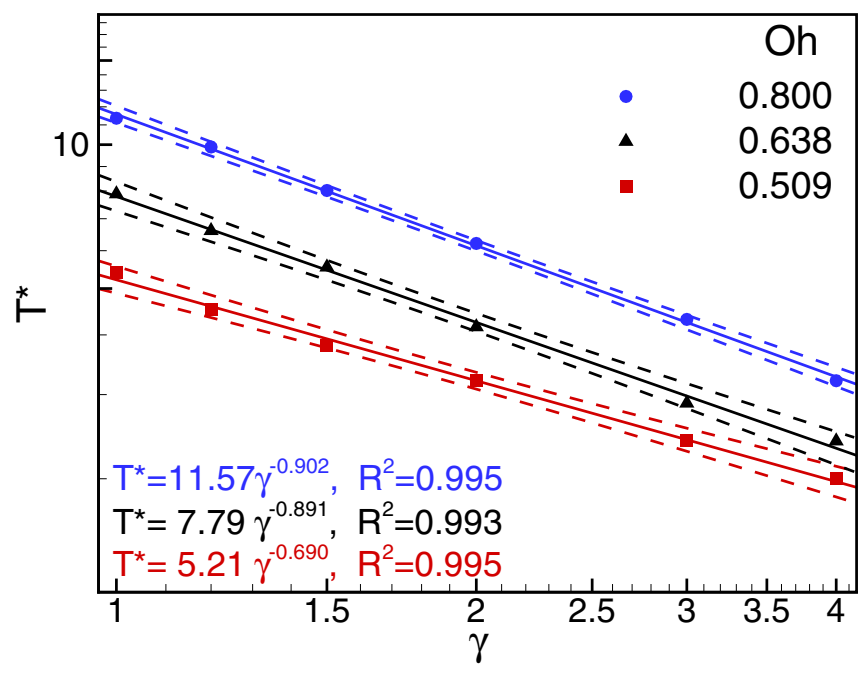

FIG. 3. Power-law temporal scaling of unequal-size microbubble coalescence with three different $\mathrm{Oh}$ numbers corresponding to cases 1, 5, and 10 in Table III. Symbols: numerical results; lines: power-law fitting.

results with the fitting lines are 0.019 and $0.999,0.02$ and 0.998 , and 0.011 and 0.999 , corresponding to $\mathrm{Oh}=0.509$, 0.638 , and 0.8 , respectively. The average difference is defined as the root-mean square of the relative error between the numerical and the fitting value. The pair of dashed lines for each case shows the $95 \%$ confidence band. From bottom up, the prefactor $\left(A_{0}\right)$ and power index $(n)$ change as the Oh number increases. As the surface tensions of these three cases remain the same $(\sigma=0.032 \mathrm{~N} / \mathrm{m})$, this result indicates that the power-law temporal scaling is affected by the liquid density and viscosity reflected in the Oh number. Closely looking into the 12 cases, we show the quantitative effects of the $\mathrm{Oh}$ number on $A_{0}$ and $n$ in Fig. 4 with symbols from the simulations. In Fig. 4(a), the prefactor $A_{0}$ shows a linear relation with $\mathrm{Oh}$ for the entire $\mathrm{Oh}$ range. The average difference of the numerical results from the fitting line is 0.02 and the corresponding correlation coefficient is 0.997 . In Fig. 4(b), the power index $n$ is linear to Oh only when $\mathrm{Oh}<$ 0.66 , with an average difference and a correlation coefficient 0.01 and 0.996 , respectively. When $\mathrm{Oh}>0.66$, the power index remains approximately a constant (0.9) with an average difference of 0.006 . The pair of dashed lines shows the $95 \%$ confidence band.

From Fig. 4, we can derive the following relationship between the normalized global coalescence time $T^{*}$ and the size inequality $\gamma$ of parent bubbles parametrized by $\mathrm{Oh}$ :

$$
T^{*}=A_{0} \gamma^{-n}
$$

TABLE III. 12 study cases varying liquid density and liquid viscosity with fixed gas density, gas viscosity, and surface tension, resulting in a range Oh number from 0.509 to 0.946 .

\begin{tabular}{lccccccccccccc}
\hline \hline Case & 1 & 2 & 3 & 4 & 5 & 6 & 7 & 8 & 9 & 10 & 11 \\
\hline Oh & 0.509 & 0.522 & 0.577 & 0.602 & 0.638 & 0.654 & 0.654 & 0.705 & 0.774 & 0.800 & 0.872 & 0.946 \\
$\rho_{h}\left(\mathrm{~kg} / \mathrm{m}^{3}\right)$ & 896 & 1408 & 1152 & 640 & 1408 & 896 & 1480 & 1152 & 640 & 896 & 1152 & 640 \\
$\eta_{h}[\mathrm{~kg} /(\mathrm{ms})]$ & 0.0122 & 0.0157 & 0.0157 & 0.0122 & 0.0191 & 0.0157 & 0.0201 & 0.0191 & 0.0157 & 0.0191 & 0.0237 & 0.0191 \\
\hline \hline
\end{tabular}



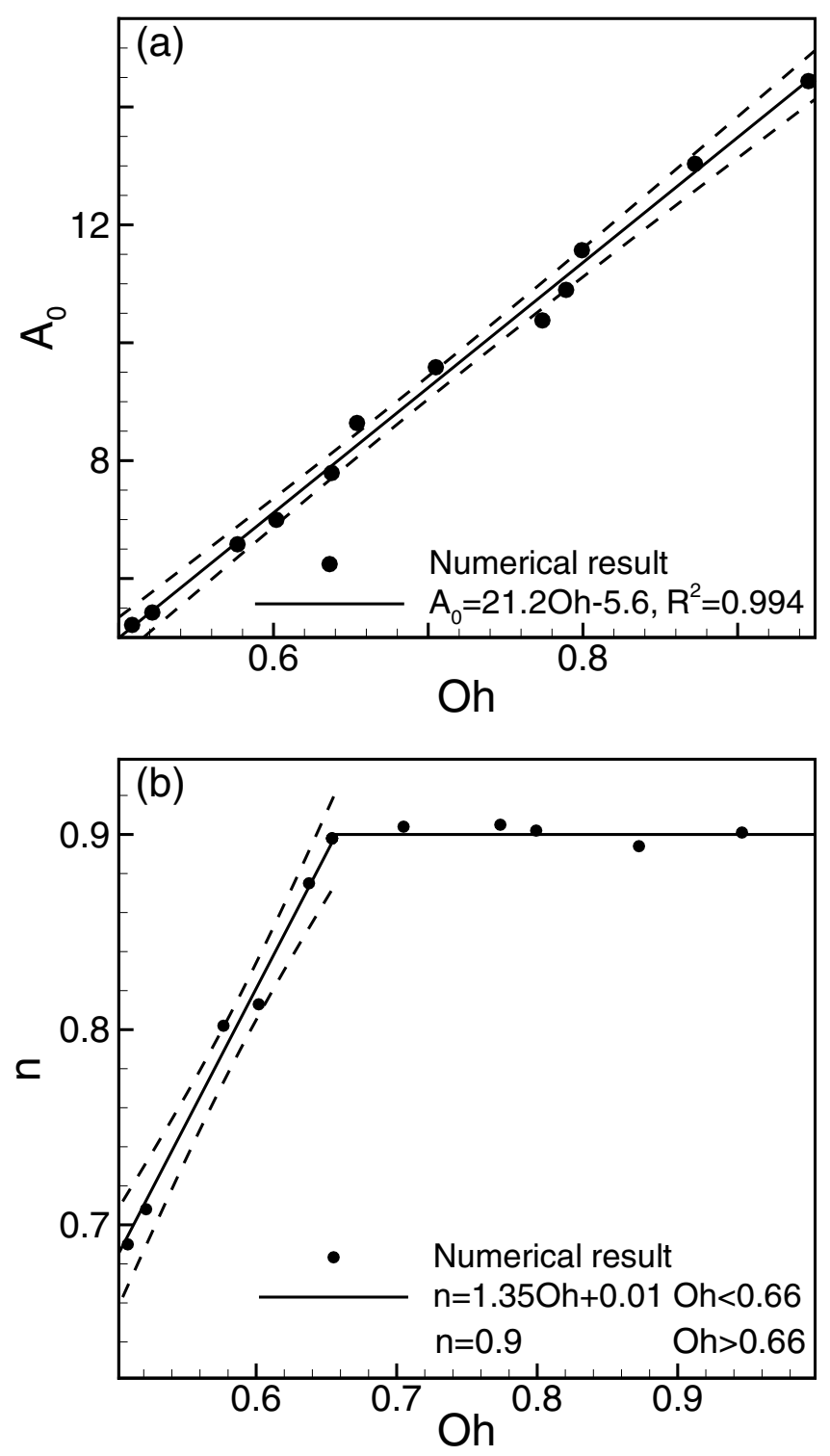

FIG. 4. Effects of Oh number on (a) prefactor $A_{0}$ and (b) power index $n$ with $R^{2}=0.983$ in the power-law temporal scaling. Symbols: numerical results; lines: fitting outcomes.

with

$$
n= \begin{cases}1.35 \mathrm{Oh}+0.01 & : 0.5<\mathrm{Oh}<0.66 \\ 0.9 & : 0.66<\mathrm{Oh}<1.0\end{cases}
$$

and $A_{0}=21.2 \mathrm{Oh}-5.6$. Equation (10) indicates the existence of a general power-law temporal scaling of microbubble coalescence in the range of $\mathrm{Oh}$ from 0.5 to 1.0 .

From Eq. (10), we obtain $T^{*}=A_{0}$ when $\gamma=1$, meaning that the prefactor of the power-law scaling represents the normalized global coalescence time of two equal-size bubbles, which is linear to Oh. The meaning of the power index $n$ is explored by $d T^{*} / d \gamma$ at $\gamma=1$. Corresponding to two ranges of Oh in Eq. (10), we have

$$
\begin{aligned}
& \left.\frac{d T^{*}}{d \gamma}\right|_{\gamma=1} \\
& = \begin{cases}28.38(\mathrm{Oh})^{2}-7.35 \mathrm{Oh}-0.06 & : 0.5<\mathrm{Oh}<0.66, \\
19.08 \mathrm{Oh}-5.04 & : 0.66<\mathrm{Oh}<1.0\end{cases}
\end{aligned}
$$

Equation (11) shows that the quickness of the coalescence from equal to unequal-size bubble coalescence is determined by Oh. These behaviors can be understood from the following aspects. First, the size inequality $\gamma$ reflects the initial driving mechanism. The driving is generated by the imbalanced surface tension forces on the parent bubbles due to the different bubble radii of both. Larger imbalance of surface tension forces generates stronger inertia, resulting in shorter global coalescence time. In our previous work [19], we have tested the effects of size inequality on the dynamics of velocity vector, pressure, and vorticity and demonstrated the effect of size inequality on the global coalescence time. It has been found that bubbles coalesce faster when the size inequality is larger. Second, when $\gamma$ is fixed, larger Oh means stronger viscous effects in the liquid, leading to longer global coalescence time. These two effects can be seen in Fig. 3. Third, when the $\mathrm{Oh}$ number is relatively large, the viscous effect from the liquid is more significant to resist the coalescence. In this case, the initially imbalanced surface tension force is less significant. Therefore, the effect of size inequality becomes negligible, resulting in a constant power index. The reasons that the transition occurs around $\mathrm{Oh}=$ 0.66 and $n$ equals to 0.90 when $0.66<\mathrm{Oh}<1$ need further investigation.

Since the temporal scaling of unequal-size microbubble coalescence has been rarely addressed in open literature, we now select 11 new and independent cases to validate the general power-law temporal scaling [Eq. (10)]. Table IV lists the new cases with combinations of fluid density, fluid viscosity, and surface tension. The 11 cases fall into three Oh numbers: $0.509,0.654$, and 0.800 .

We plot the simulation results of global coalescence time $\left(T^{*}\right)$ versus size inequality $\gamma$ in Fig. 5 for all the cases grouped by three Oh numbers in (a), (b), and (c), respectively.

TABLE IV. 11 new and independent cases with three Oh numbers of $0.509,0.654$, and 0.800 for validation of Eq. (10).

\begin{tabular}{lcccccccccccc}
\hline \hline Case & 13 & 14 & 15 & 16 & 17 & 18 & 19 & 20 & 21 & 22 \\
\hline Oh & 0.509 & 0.509 & 0.509 & 0.509 & 0.654 & 0.654 & 0.654 & 0.800 & 0.800 & 0.800 & 0.800 \\
$\rho_{h}\left(\mathrm{~kg} / \mathrm{m}^{3}\right)$ & 1197 & 896 & 1000 & 680 & 896 & 448 & 640 & 600 & 680 & 1482 & 896 \\
$\eta_{h}[\mathrm{~kg} /(\mathrm{m} \mathrm{s})]$ & 0.0122 & 0.0149 & 0.0182 & 0.0160 & 0.0111 & 0.0157 & 0.0107 & 0.0111 & 0.0204 & 0.0371 & 0.0271 \\
$\sigma(\mathrm{N} / \mathrm{m})$ & 0.024 & 0.048 & 0.064 & 0.073 & 0.016 & 0.064 & 0.021 & 0.016 & 0.048 & 0.064 & 0.073 \\
\hline \hline
\end{tabular}



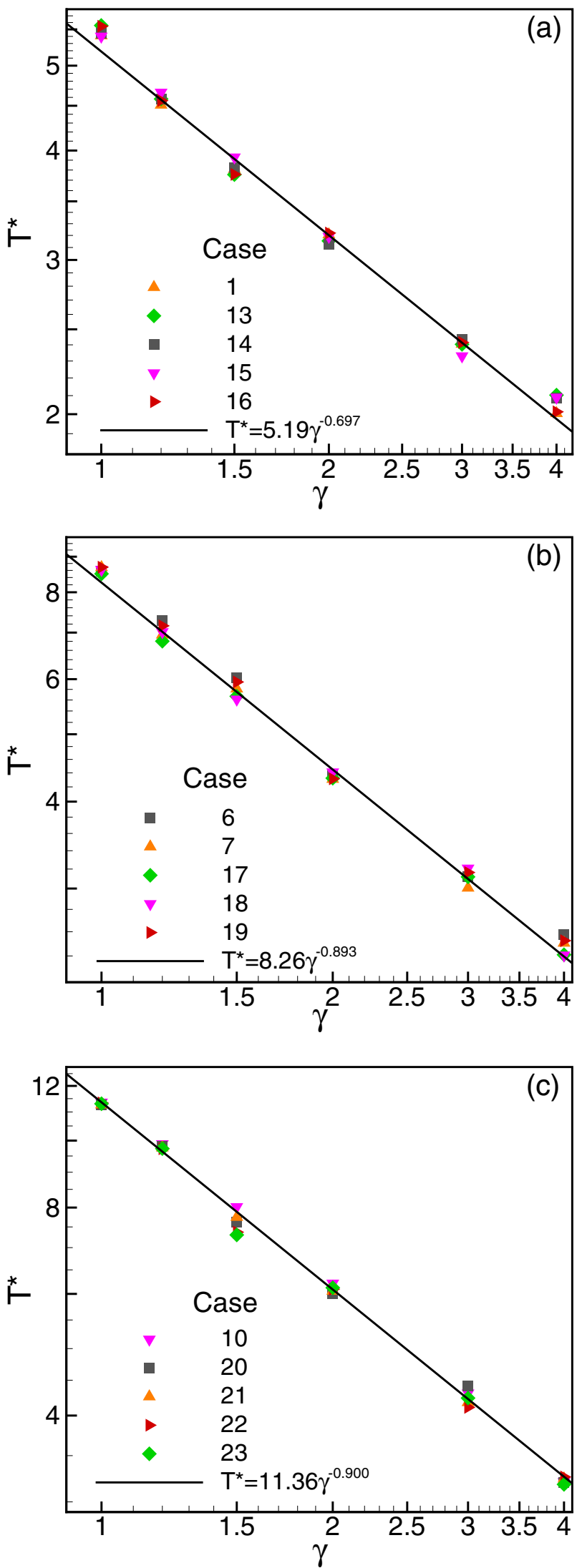

FIG. 5. General power-law temporal scaling for unequal-size air microbubble coalescence at $\mathrm{Oh}=0.509(\mathrm{a}), 0.654(\mathrm{~b})$, and $\mathrm{Oh}=0.8$ (c). Symbols: simulation results. Lines: general power-law [Eq. (10)] prediction.
The symbols are simulation results and the lines are from Eq. (10). Corresponding to $\mathrm{Oh}=0.509,0.654$, and 0.800 , Eq. (10) predicts the power index of the power-law temporal scaling as $0.692,0.893$, and 0.900 , respectively. The simulation results agree well with the predictions in each of the three evaluations. Thus, Eq. (10) is regarded as a general power-law temporal scaling of unequal-size microbubble coalescence.

\section{SUMMARY}

We have systematically studied global coalescence of unequal-size microbubbles characterized by Oh number using lattice Boltzmann simulation. The validation was on the temporal scaling of the early stage (neck growth) of equal-size bubble coalescence. The simulation results have well captured the half-power scaling of the neck growth from analytical prediction. Thanks to the significant acceleration of GPU parallelism for LBM, we were able to conduct 138 simulations in 23 cases varying the liquid density, liquid viscosity, and surface tension from $448 \sim 1482\left(\mathrm{~kg} / \mathrm{m}^{3}\right), 0.011 \sim$ $0.0237[\mathrm{~kg} /(\mathrm{m} \mathrm{s})]$, and $0.024 \sim 0.073(\mathrm{~N} / \mathrm{m})$, respectively. The Oh number ranges from 0.5 to 1.0. The effects of liquid viscosity, liquid density, and surface tension, characterized by Oh number, have been investigated with the following three results:

(1) The power-law temporal scaling of unequal-size microbubble coalescence $T^{*}=A_{0} \gamma^{-n}$ generally exists in the range of $0.5<\mathrm{Oh}<1.0$, where no damping oscillation is involved in the post coalescence.

(2) The prefactor $A_{0}$ is linear to $\mathrm{Oh}$ in the range of 0.5 to 1.0 .

(3) The power index $n$ is linear to $\mathrm{Oh}$ in the range of 0.5 to 0.66 and remains constant in the range of 0.66 to 1.0.

A general power-law temporal scaling of normalized global coalescence vs size inequality has been derived. The physical understanding of both prefactor and power index have been explored. Additionally, 11 new and independent cases that vary the fluid density, fluid viscosity, and surface tension are grouped by three Oh numbers, $0.509,0.654$, and 0.800 . The simulation results of these 11 cases agree well with the predictions by Eq. (10), demonstrating the reliability of the general power-law temporal scaling in the specified Oh range. This scaling was obtained when the gas phase was fixed. Based on recent studies [8,13,47], the droplet coalescence is sensitive to the outer fluid (heavy fluid) but insensitive to the inner fluid (light fluid). We believe that the results of this work obtained from a fixed gas (air) are applicable for different gases.

The general power-law temporal scaling for unequal-size microbubble coalescence is revealed for microbubble coalescence. This temporal scaling can be useful for various engineering and medical applications as microbubble transport frequently appears in different microfluidic systems and bubble coalescence commonly occurs during the transport. It is known that studying global bubble coalescence in microfluidic systems can be challenging. In experiments, it requires high-quality and fast-speed cameras. And, in computation, it requires advanced modeling and fast computation speed. Equation (10) can be used to predict the bubble coalescence 
time based on the Oh number and the size inequality of parent bubbles when the Oh number falls in the specified range. Base on our reference survey of open resources, many liquid-gas systems have the Oh number in the specified range of Eq. (10). We hope this general power-law temporal scaling can further support effective and optimal design and fabrication of realworld microfluidic systems.

\section{ACKNOWLEDGMENTS}

This research is supported by the National Science Foundation under Grant No. 1264739. And this work used the Extreme Science and Engineering Discovery Environment (XSEDE), which is supported by National Science Foundation Grant No. ACI-1548562.
[1] M. K. H. AL-Mashhadani, J. S. Wilkinson, and B. W. Zimmerman, Airlift bioreactor for biological applications with microbubble mediated transport processes, Chem. Eng. Sci. 137, 243 (2015).

[2] N. Matsuki, S. Ichiba, T. Ishikawa, O. Nagano, M. Takeda, Y. Ujike, and T. Yamaguchi, Blood oxygenation using microbubble suspensions, Eur. Biophys. J. 41, 571 (2012).

[3] K. Terasaka, A. Hirabayashi, T. Nishino, S. Fujioka, and D. Kobayashi, Development of microbubble aerator for waste water treatment using aerobic activated sludge, Chem. Eng. Sci. 66, 3172 (2011).

[4] P. Dahiya, M. Caggioni, and P. T. Spicer, Arrested coalescence of viscoelastic droplets: Polydisperse doublets, Philos. Trans. R. Soc. London A 374, 20150132 (2016).

[5] S. Ghaemi, P. Rahimi, and D. S. Nobes, The effect of gasinjector location on bubble formation in liquid cross flow, Phys. Fluids 22, 043305 (2010).

[6] J. Eggers, J. R. Lister, and H. A. Stone, Coalescence of liquid drops, J. Fluid Mech. 401, 293 (1999).

[7] J. D. Paulsen, J. C. Burton, and S. R. Nagel, Viscous to Inertial Crossover in Liquid Drop Coalescence, Phys. Rev. Lett. 106, 114501 (2011).

[8] J. D. Paulsen, R. Carmigniani, A. Kannan, J. C. Burton, and S. R. Nagel, Coalescence of bubbles and drops in an outer fluid, Nat. Commun. 5, 3182 (2014).

[9] R. Chen, H. Yu, and L. Zhu, Effects of initial conditions on the coalescence of micro-bubbles, Proc. Inst. Mech. Eng., Part C 232, 457 (2017).

[10] C. R. Anthony, P. M. Kamat, S. S. Thete, J. P. Munro, J. R. Lister, M. T. Harris, and O. A. Basaran, Scaling laws and dynamics of bubble coalescence, Phys. Rev. Fluids 2, 083601 (2017).

[11] C. A. Miller and L. E. Scriven, The oscillations of a fluid droplet immersed in another fluid, J. Fluid Mech. 32, 417 (1968).

[12] O. A. Basaran, Nonlinear oscillations of viscous liquid drops, J. Fluid Mech. 241, 169 (1992).

[13] R. L. Stover, C. W. Tobias, and M. M. Denn, Bubble coalescence dynamics, AIChE J. 43, 2385 (1997).

[14] R. Chen, J. Zeng, and H. Yu, Mechanism of damped oscillation in microbubble coalescence, Comput. Fluids 183, 38 (2019).

[15] A. Agarwal, W. J. Ng, and Y. Liu, Principle and applications of microbubble and nanobubble technology for water treatment, Chemosphere 84, 1175 (2011).

[16] Y. H. Qian, Fractional propagation and the elimination of staggered invariants in lattice-BGK models, Int. J. Mod. Phys. C 08, 753 (1997).

[17] L. Zhu, N. Kroodsma, J. Yeom, J. L. Haan, M. A. Shannon, and D. D. Meng, An on-demand microfluidic hydrogen generator with self-regulated gas generation and self-circulated reactant exchange with a rechargeable reservoir, Microfluid. Nanofluid. 11, 569 (2011).

[18] S. Zhou, Y. Cao, R. Chen, T. Sun, K. Fezzaa, H. Yu, and L. Zhu, Study on coalescence dynamics of unequal-sized microbubbles captive on solid substrate, Exp. Therm. Fluid Sci. 98, 362 (2018).

[19] R. Chen, H. Yu, L. Zhu, L. Taehun, and R. Patil, Spatial and temporal scaling of unequal microbubble coalescence, AIChE J. 63, 1441 (2017).

[20] H. D. Chen, S. Y. Chen, and W. H. Matthaeus, Recovery of the Navier-Stokes equations using lattice-gas Boltzmann method, Phys. Rev. A 45, R5339 (1992).

[21] Y. H. Qian, D. Dhumieres, and P. Lallemand, Lattice Boltzmann model for Navier-Stokes equation, Europhys. Lett. 17, 479 (1992).

[22] M. R. Swift, W. R. Osborn, and J. M. Yeomans, Lattice Boltzmann Simulation of Nonideal Fluids, Phys. Rev. Lett. 75, 830 (1995).

[23] M. R. Swift, E. Orlandini, W. R. Osborn, and J. M. Yeomans, Lattice Boltzmann simulations of liquid-gas and binary fluid systems, Phys. Rev. E 54, 5041 (1996).

[24] X. Y. He, X. Shan, and G. D. Doolen, Discrete Boltzmann equation model for nonideal gases, Phys. Rev. E 57, R13 (1998).

[25] X. He, S. Chen, and R. Zhang, A lattice Boltzamnn scheme for incompressible multiphase flow and its application in simulation of Rayleigh-Taylor instability, J. Comput. Phys. 152, 642 (1999).

[26] J. W. Cahn and J. E. Hilliard, Free energy of a nonuniform system. I. Interfacial free energy, J. Chem. Phys. 28, 258 (1958).

[27] D. M. Anderson, G. B. McFadden, and A. A. Wheeler, Diffusinterface method in fluid mechanics, Annu. Rev. Fluid Mech. 30, 139 (1998).

[28] D. Jacqmin, Calculation of two-phase Navier-Stokes flows using phase-field modeling, J. Comput. Phys. 155, 96 (1999).

[29] T. Lee and C.-L. Lin, A stable discretization of the lattice Boltzmann equation for simulation of incompressible two-phase flows at high density ratio, J. Comput. Phys. 206, 16 (2005).

[30] T. Lee and P. F. Fischer, Eliminating parasitic currents in the lattice Boltzmann equation method for nonideal gases, Phys. Rev. E 74, 046709 (2006).

[31] T. Lee, Effects of incompressibility on the elimination of parasitic currents in the lattice Boltzmann equation method for binary fluids, Comput. Math. Appl. 58, 987 (2009).

[32] T. Lee and L. Liu, Lattice Boltzmann simulations of micronscale drop impact on dry surfaces, J. Comput. Phys. 229, 8045 (2010).

[33] M. J. Mawson and A. J. Revell, Memory transfer optimization for a lattice Boltzmann solver on Kepler architecture nVidia GPUs, Comput. Phys. Commun. 185, 2566 (2014). 
[34] O. Navarro-Hinojosa, S. Ruiz-Loza, and M. AlencastreMiranda, Physically based visual simulation of the lattice Boltzmann method on the GPU: A survey, J. Supercomput. 74, 3441 (2018).

[35] S. P. Vanka, A. F. Shinn, and K. C. Sahu, Computational fluid dynamics using graphics processing units: challenges and opportunities, in ASME 2011 International Mechanical Engineering Congress and Exposition (American Society of Mechanical Engineers, New York, 2011), pp. 429-437.

[36] P. R. Redapangu, K. Chandra Sahu, and S. P. Vanka, A study of pressure-driven displacement flow of two immiscible liquids using a multiphase lattice Boltzmann approach, Phys. Fluids 24, 102110 (2012).

[37] T. C. Huang, C. Y. Chang, and C. A. Lin, Simulation of droplet dynamic with high density ratio two-phase lattice boltzmann model on multi-GPU cluster, Comput. Fluids 173, 80 (2018).

[38] N. Chen and H. Yu, Mechanism of axis switching in low aspect-ratio rectangular jets, Comput. Math. Applicat. 67, 437 (2014).

[39] Z. Wang, N. Chen, Y. Zhao, and H. Yu, GPU-accelerated lattice Boltzmann method for extracting real biomechanical geometry and volumetric boundary condition, Comput. Fluids 115, 192 (2015).
[40] S. An, H. Yu, Z. Wang, R. Chen, B. Kapadia, and J. Yao, Unified mesoscopic modeling and GPU-accelerated computational method for image-based pore-scale porous media flows, Int. J. Heat Mass Transfer 115, 1192 (2017).

[41] S. An, H. Yu, and J. Yao, GPU-accelerated volumetric lattice Boltzmann method for porous media flow, J. Pet. Sci. Eng. 156, 546 (2017).

[42] J. Towns, T. Cockerill, M. Dahan, I. Foster, K. Gaither, A. Grimshaw, V. Hazlewood, S. Lathrop, D. Lifka, G. D. Peterson et al., XSEDE: accelerating scientific discovery, Comput. Sci. Eng. 16, 62 (2014).

[43] J. S. Rowlinson and B. Widom, Molecular Theory of Capillarity (Oxford Univiversity Press, Oxford, UK, 1989).

[44] D. Jamet, O. Lebaigue, N. Coutris, and J. M. Delhaye, The second gradient method for the direct numerical simulation of liquid-vapor flows with phase change, J. Comput. Phys. 169, 624 (2001).

[45] H. P. Kavehpour, Coalescence of drops, Annu. Rev. Fluid Mech. 47, 245 (2015)

[46] H. Aryafar and H. P. Kavehpour, Drop coalescence through planar surfaces, Phys. Fluids 18, 072105 (2006).

[47] J. P. Munro, C. R. Anthony, O. A. Basaran, and J. R. Lister, Thin-sheet flow between coalescing bubbles, J. Fluid Mech. 773, R3 (2015). 